\title{
ARTICLE OPEN Experimental demonstration of quantum finite automaton
}

\author{
Yuling Tian $\mathbb{D}^{1}$, Tianfeng Feng $\mathbb{D}^{1}$, Maolin Luo $\mathbb{D}^{1}$, Shenggen Zheng $\mathbb{D}^{2,3}$ and Xiaoqi Zhou ${ }^{1}$
}

In quantum information science, a major task is to find the quantum models that can outperform their classical counterparts. Automaton is a fundamental computing model that has wide applications in many fields. It has been shown that the quantum version of automaton can solve certain problem using a much smaller state space compared to the classical automaton. Here we report an experimental demonstration of an optical quantum automaton, which is used to solve the promise problems of determining whether the length of an input string can be divided by a prime number $P$ with no remainder or with a remainder of $R$. Our quantum automaton can solve such problem using a state space with only three orthonormal states, whereas the classical automaton needs no less than $P$ states. Our results demonstrate the quantum benefits of a quantum automaton over its classical counterpart and paves the way for implementing quantum automaton for more complicated and practical applications.

npj Quantum Information (2019)5:56; https://doi.org/10.1038/s41534-019-0163-x

\section{INTRODUCTION}

Quantum information science is a research field focused on enhancing problem-solving efficiency by utilizing quantum effects. The most prominent example is quantum computer-by changing the basic elements of a computer from bits and logic gates to quantum bits and quantum logic gates, quantum computer can achieve an exponential speedup in solving certain problems, including factoring ${ }^{1}$ and simulation of complex systems. $^{2-4}$ Extensive efforts have been made to build such computing machine and small-scale quantum computers have already been constructed in different physical systems. ${ }^{5-11} \mathrm{~A}$ series of quantum algorithms have been demonstrated on these prototype machines to show the quantum benefits in tackling particular problems. ${ }^{12-16}$

Computer is not the only device whose capability can be boosted by introducing quantum elements. Finite automaton (FA) is another good example. As one of the most fundamental models in computer science, FA can be used to model problems in many fields, including mathematics, artificial intelligence, games or linguistics. ${ }^{17,18}$ The concept of quantum finite automaton (QFA) was invented as the quantum version of FA by Kondacs and Watrous $^{19}$ and also by Moore and Crutchfield. ${ }^{20}$ It has been theoretically predicted that QFA can solve certain problems more efficiently than its classical counterpart. ${ }^{21,22}$ However, to the best of our knowledge, no experiment has been performed to prove the quantum benefits of QFA yet.

In this letter, we have built a proof-of-principle optical QFA that can solve some certain problems more space-efficiently than a classical FA. In our experiment, we have proven that a QFA composed with a three-dimensional quantum state suffice to solve a promise problem whereas a classical FA needs much larger state space to solve the same problem.

The paper is structured as follows. We will first introduce some background knowledge about FA and QFA. Then we will explain how to use a QFA to solve a certain promise problem. At last, we will present our experimental demonstration of an optical QFA solving a promise problem.

\section{RESULTS}

A deterministic finite automaton (DFA) is a finite-state machine that accepts or rejects input strings of symbols. Here deterministic refers to the fact that a DFA only produces a unique computation for each input string. We only consider the case of DFAs as it has been proven that DFAs have equivalent computing power to nondeterministic finite automata. ${ }^{17,23,24}$

As shown in Fig. 1a, a typical DFA is illustrated with a state diagram. In this example automaton, there are two states $S_{0}$ and $S_{1}$, which are denoted graphically by circles. The automaton takes a finite sequence of $0 \mathrm{~s}$ and $1 \mathrm{~s}$ as input. For each state, there is a transition arrow leading out to a next state for both 0 and 1. Upon reading a symbol, a DFA jumps deterministically from one state to another by following the transition arrow. For example, if the automaton is currently in state $S_{0}$ and the current input symbol is 1 , then it deterministically jumps to state $S_{1}$. A DFA has a start state (denoted graphically by an arrow coming in from nowhere) where computations begin, and a set of accepted states (denoted graphically by a double circle). If the automaton ends at an accepted state after reading the last symbol of the input string, this string of symbols is regarded as being accepted by the DFA; otherwise, it is regarded as being rejected by the DFA. DFAs are often used to solve (recognize) decision problems, which are defined as computational problems where the answer for every instance is either Yes or No. For example, determining whether a binary number is even or not is a typical decision problem, which can be solved by the DFA shown in Fig. 1a.

QFA, the quantum version of DFA, is realized by changing the basic elements of DFA to the quantum components. As shown in Fig. $1 \mathrm{~b}$, inside a QFA sits a quantum state $|\psi\rangle$ with $m$ orthonormal basis states $|0\rangle,|1\rangle, \ldots,|m-2\rangle,|m-1\rangle$. One or several of the $m$

\footnotetext{
${ }^{1}$ School of Physics, State Key Laboratory of Optoelectronic Materials and Technologies, Sun Yat-sen University, 510006 Guangzhou, China; ${ }^{2}$ Peng Cheng Laboratory, 518000 Shenzhen, China and ${ }^{3}$ Institute for Quantum Science and Engineering, Southern University of Science and Technology, 518055 Shenzhen, China Correspondence: Shenggen Zheng (zhengsg@sustc.edu.cn) or Xiaoqi Zhou (zhouxq8@mail.sysu.edu.cn)

These authors contributed equally: Yuling Tian, Tianfeng Feng
}

Received: 5 January 2019 Accepted: 21 May 2019

Published online: 11 June 2019 

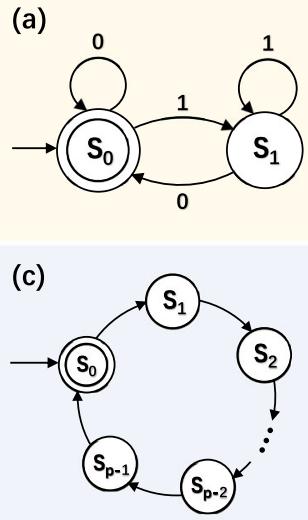

(b)

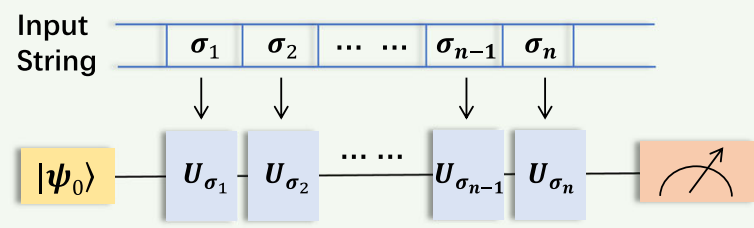

(d)

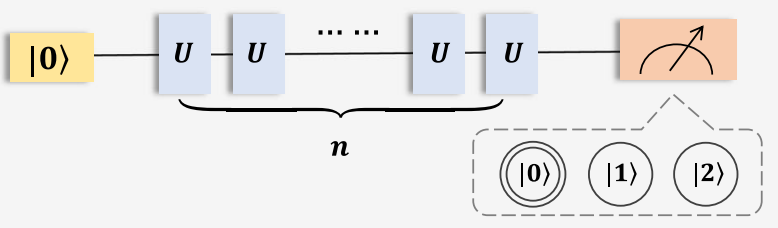

Fig. 1 State diagrams of deterministic finite automata (DFAs) and quantum finite automata (QFAs). a The state diagram of a typical DFA. $S_{0}$ and $S_{1}$ are the two possible states of the DFA, where $S_{0}$ is both the start state and the accepted state. For each state, there is a transition arrow leading out to a next state for both 0 and 1 . Upon reading a symbol ( 0 or 1$)$, the DFA will jump deterministically from one state to another following the transition arrow. The current DFA accepts only binary numbers that are multiples of 2. $\mathbf{b}$ The state diagram of a general QFA. Inside the QFA is an $m$-dimensional quantum state, with $\left|\psi_{0}\right\rangle$ as the start state and $|0\rangle,|1\rangle, \ldots,|m-2\rangle,|m-1\rangle$ being its $m$ orthonormal basis states, where some of these basis states are designated as accepted states. The input string $\sigma_{1} \sigma_{2} \cdots \sigma_{n-1} \sigma_{n}$ determines how the quantum state will evolve. Every time a symbol $\sigma_{i}$ is read in, a corresponding unitary operator $U_{\sigma_{i}}$ is applied on the quantum state. After reading the last symbol of the string, the final quantum state would be measured and thus projected to one of the $m$ basis states. The input string would then be accepted or rejected by the QFA according to whether or not the quantum state is projected to one of the accepted states. c A DFA for solving a promise problem. This DFA can be used to determine whether the length of an input string is an integer multiple of a prime number $P$ or not. d A QFA for solving the same promise problem. Inside the DFA is a three-dimensional quantum state, with $|0\rangle,|1\rangle$, and $|2\rangle$ being its 3 orthonormal basis states, where $|0\rangle$ is both the start state and the only accepted state. The quantum state will go through $n$ copies of unitary operator $U$, where $n$ is the length of the input string

basis states are designated as accepted states. Before the computation starts, the QFA rests at a starting state $\left|\psi_{0}\right\rangle$. The computation of a QFA with an input string $\sigma_{1} \sigma_{2} \ldots \sigma_{n-1} \sigma_{n}\left(\sigma_{i}\right.$ is the ith symbol of the string) works as follows: The QFA reads in the string from left to right, symbol by symbol. Upon reading a symbol $\sigma_{i}$, a corresponding unitary operator $U_{\sigma_{i}}$ is applied on the state. After reading the last symbol of the string, the final state of the QFA would be $\left|\psi_{n}\right\rangle=U_{\sigma_{n}} U_{\sigma_{n-1}} \cdots U_{\sigma_{2}} U_{\sigma_{1}}\left|\psi_{0}\right\rangle$. A projective measurement in the basis of $|0\rangle,|1\rangle, \ldots,|m-2\rangle,|m-1\rangle$ is then applied on $\left|\psi_{n}\right\rangle$ and the state would be projected to one of the $m$ basis states. If the state is projected to an accepted state, the string is regarded as being accepted by the QFA; otherwise the string is rejected.

To show the quantum benefits of QFA over DFA, we now consider a particular problem. Assuming the length of an input string is guaranteed to be either $k^{*} P$ or $k^{*} P+R$, where $P$ is a prime number, $R$ is a positive integer less than $P$, and $k$ is an arbitrary integer no less than 0 . The task is to determine whether the length of the string can be divided by $P$ with no remainder or with a remainder of $R$. Such task is a promise problem, ${ }^{25}$ which is a generalization of decision problem, where the input is promised to belong to a particular subset of all possible inputs. Let us show how to solve this problem with a DFA first. As shown in Fig. 1c, there are $P$ states in the diagram and $S_{0}$ is both the start and only accepted state in the DFA. Here symbols with different alphabets are treated the same way as the concrete alphabets of symbols do not affect the length of the string. Therefore, the labels besides the transition arrows are omitted here. The $P$ states and the transition arrows form a circle in the diagram, which means the DFA will change back to its original state after $k * P$ moves. As a result, if the length of the string is $k^{*} P$, the DFA, which starts at $S_{0}$, will also end at $S_{0}$, signifying the acceptance of the input string; if the length is $k^{*} P+R$, the DFA will end at $S_{R}$ and the input string would be rejected. Although the DFA can only end at either of the two states $S_{0}$ or $S_{R}$ here, its state space cannot be squeezed. It has been proven that, to solve this problem, one needs a DFA with no less than $P$ states. ${ }^{22}$

We now explain how to solve this problem with a QFA, using a quantum state space with just three orthonormal basis states. As shown in Fig. 1d, inside the QFA is a 3-dimensional quantum state, namely a qutrit, with three orthonormal basis states $|0\rangle,|1\rangle$, and $|2\rangle$. The start state of the QFA is set as $|0\rangle$. Just as the DFA, symbols with different alphabets are treated the same way here as well. Upon reading in a symbol, no matter what the symbol is, the same unitary $U$ would be applied to the quantum state. As a result, after reading in the last symbol of the input string, the final quantum state would be either $U^{k^{*} P}|0\rangle$ or $U^{k^{*} P}+R|0\rangle$. By properly choosing unitary operator $U$, these two states can be orthogonal to each other. We will set the unitary operator $U=U_{e}^{\dagger} U_{c} U_{e}$, where both of $U_{\mathrm{e}}$ and $U_{\mathrm{c}}$ are three-dimensional unitary operators. $U_{\mathrm{c}}$ is defined as

$U_{c}=\left(\begin{array}{ccc}1 & 0 & 0 \\ 0 & \cos \frac{2 \pi Q}{P} & -\sin \frac{2 \pi Q}{P} \\ 0 & \sin \frac{2 \pi Q}{P} & \cos \frac{2 \pi Q}{P}\end{array}\right)$,

in which $Q$ is a positive integer less than $P$. Here $Q$ is properly chosen to ensure that the inequality $\cos \left(\frac{2 \pi Q}{P} R\right)<0$ holds. For given $P$ and $R$, if $\frac{P}{4} \leq R \leq \frac{3 P}{4}, Q$ is set to be 1 ; if $R<\frac{P}{4}, Q$ is set to be $\left[\frac{P}{4 R}\right]$; if $R$ gt; $\frac{3 P}{4}, Q$ is set to be $\left\lfloor\frac{P}{R}\left(j+\frac{1}{4}\right)\right\rfloor+1$, where $j$ is an integer such that $\frac{1}{4}<j \frac{P-R}{R}-\left\lfloor j \frac{P-R}{R}\right\rfloor<\frac{2}{3}$. 22 It can be verified that

$U_{c}^{k * P}=\left(\begin{array}{lll}1 & 0 & 0 \\ 0 & 1 & 0 \\ 0 & 0 & 1\end{array}\right)$

and

$U_{c}^{k^{*} P+R}=\left(\begin{array}{ccc}1 & 0 & 0 \\ 0 & t & -\sqrt{1-t^{2}} \\ 0 & \sqrt{1-t^{2}} & t\end{array}\right)$,

where $t=\cos \left(\frac{2 \pi Q}{P} R\right)$. $U_{\mathrm{e}}$ is defined as

$U_{e}=\left(\begin{array}{ccc}\sqrt{\frac{-t}{1-t}} & \sqrt{\frac{1}{1-t}} & 0 \\ \sqrt{\frac{1}{1-t}} & -\sqrt{\frac{-t}{1-t}} & 0 \\ 0 & 0 & 1\end{array}\right)$ 


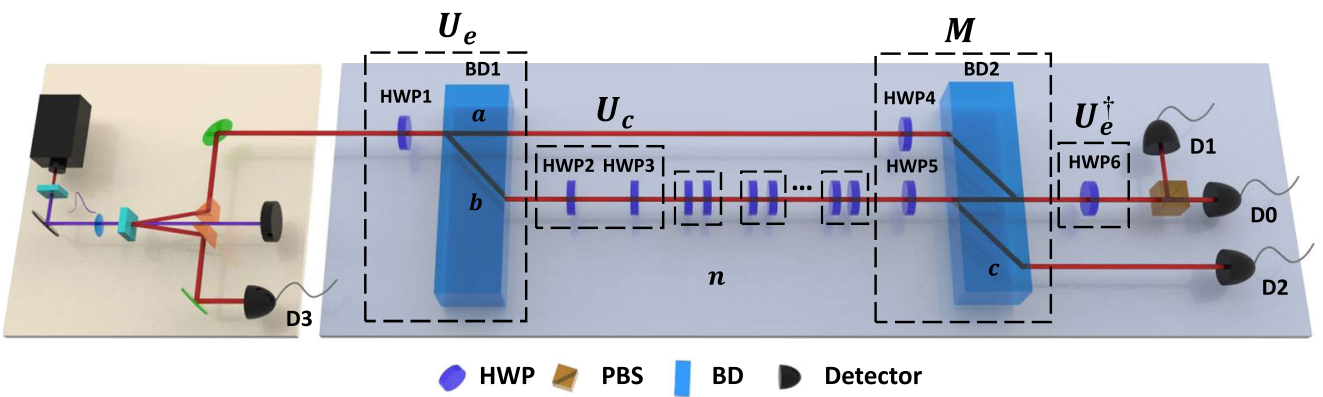

Fig. 2 Experimental setup of an optical quantum finite automaton (QFA). The experimental setup is used to implement a QFA for determining whether the length of the string $n$ is $k^{*} P$ or $k^{*} P+R$, where $P$ is a prime number, $R$ is a positive integer less than $P$ and $k$ is an arbitrary integer no less than 0 . With $(P, R)$ setting as $(3,1),(3,2),(5,1)$, or $(5,2)$, four different QFAs are built accordingly. A photon pair with central wavelength at $780 \mathrm{~nm}$ is generated by pumping a type-II beta barium borate (BBO) crystal with a UV laser. By detecting a photon at detector 3 , a heralded single photon, working as a qutrit in the QFA, is created and led into the optical circuit. The photon qutrit, encoded in both the polarization and the path degree of freedom, passes through the unitary operators $U_{\mathrm{e}}, U_{\mathrm{c}}^{n}, M$ and $U_{\mathrm{e}}^{\dagger}$ in turn. $U_{\mathrm{e}}$ is realized by a half-waveplate (HWP1) and a beam displacer (BD1). HWP2 and HWP3 together realize a $U_{c} . U_{c}^{n}$ is realized by cascading $n$ copies of such $U_{c} . M_{1}$ composed of HWP4 (at $45^{\circ}$ ), HWP5 (at $45^{\circ}$ ) and BD2, maps $V_{\mathrm{a}}$ to $H_{\mathrm{b}}, H_{\mathrm{b}}$ to $V_{\mathrm{b}}$ and $V_{\mathrm{b}}$ to $H_{\mathrm{c}}$, where $H$ and $V$ denote horizontal and vertical polarizations and $a, b$, and $c$ denote the paths of the photon. By redefining the basis states, the qutrit, written in the form of the expansion of the basis states, would remain unchanged after passing through $M . U_{\mathrm{e}}^{\dagger}$ is realized by a single half-waveplate (HWP6). The qutrit is at last measured and projected to the basis states

(a)

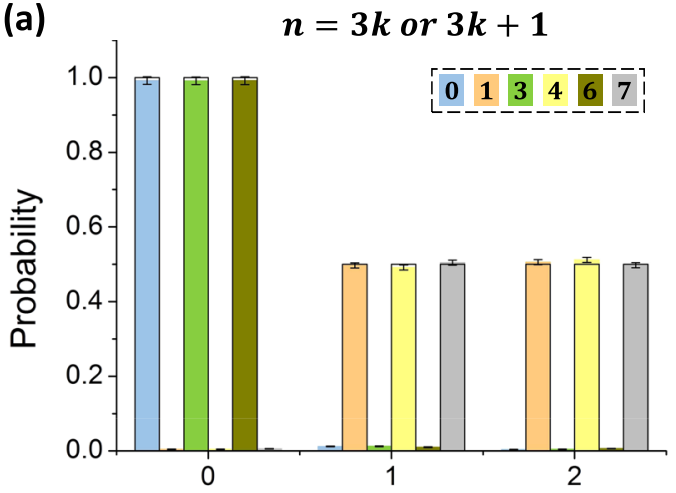

(c)

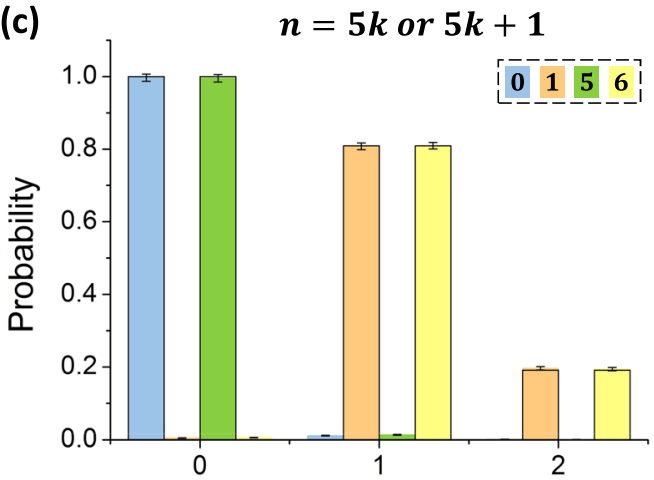

(b)

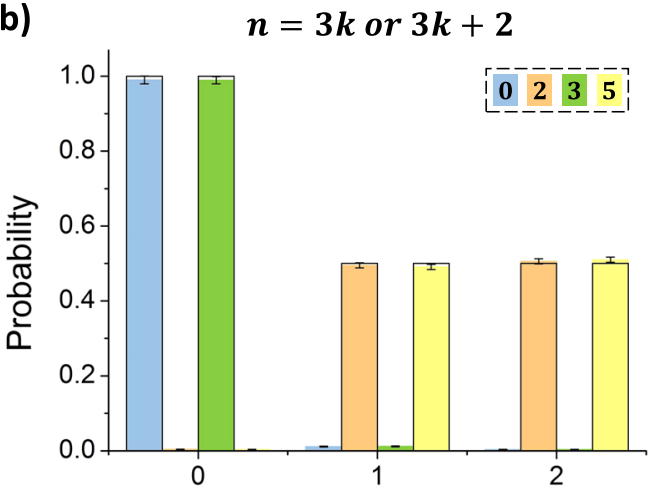

(d)

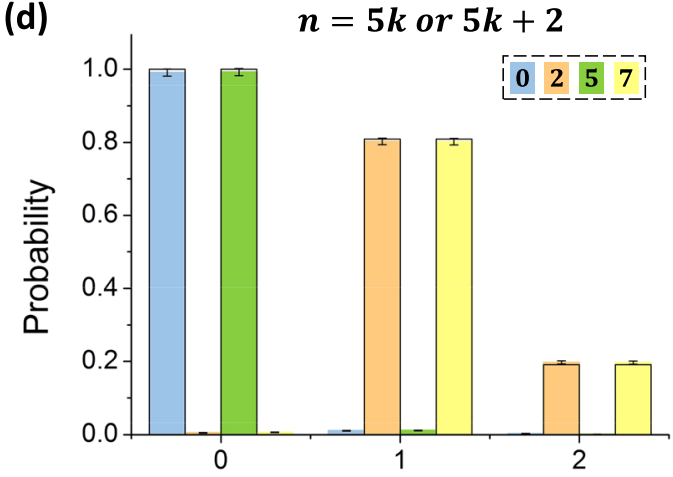

Fig. 3 Experimental results for four different quantum finite automata (QFAs). 0, 1, and 2 on the $x$ axis denote photon detections on D0, D1, and D2, respectively. a The results of QFA for determining whether the length of the input string is $3 k$ or $3 k+1$. The QFA can distinguish between the two sets $\{0,3,6\}$ and $\{1,4,7\}$ with an average success rate of $99.007 \pm 0.041 \%$. b The results of QFA for determining whether the length of the input string is $3 k$ or $3 k+2$. The QFA can distinguish between the two sets $\{0,3\}$ and $\{2,5\}$ with an average success rate of $99.075 \pm 0.048 \%$. c The results of QFA for determining whether the length of the input string is $5 k$ or $5 k+1$. The QFA can distinguish between the two sets $\{0,5\}$ and $\{1,6\}$ with an average success rate of $99.079 \pm 0.048 \%$. d The results of QFA for determining whether the length of the input string is $5 k$ or $5 k+2$. The QFA can distinguish between the two sets $\{0,5\}$ and $\{2,7\}$ with an average success rate of $99.077 \pm 0.047 \%$.The error bars (SD) are calculated according to Poissonian counting statistics 


\section{4}

and $U_{\mathrm{e}}^{2}=I$. It can converts $|0\rangle$ to $\left(\sqrt{\frac{-t}{1-t}}, \sqrt{\frac{1}{1-t}}, 0\right)^{T}$ and also convert $\left(\sqrt{\frac{-t}{1-t}}, \sqrt{\frac{1}{1-t}}, 0\right)^{T}$ back to $|0\rangle$. Putting them together, one gets $U^{k^{*} P}|0\rangle=|0\rangle$ and $U^{k * P+R}|0\rangle=\sqrt{-t}|1\rangle+\sqrt{1+t}|2\rangle$ which are derived from the following calculations

$$
\begin{aligned}
& U_{\mathrm{e}}^{\dagger} U_{\mathrm{c}}^{k * P} U_{\mathrm{e}}|0\rangle=U_{\mathrm{e}}^{\dagger} U_{\mathrm{e}}|0\rangle=|0\rangle, \\
& U_{\mathrm{e}}^{\dagger} U_{\mathrm{c}}^{k * P+R} U_{\mathrm{e}}|0\rangle \\
& =\left(\begin{array}{ccc}
\sqrt{\frac{-t}{1-t}} & \sqrt{\frac{1}{1-t}} & 0 \\
\sqrt{\frac{1}{1-t}} & -\sqrt{\frac{-t}{1-t}} & 0 \\
0 & 0 & 1
\end{array}\right)\left(\begin{array}{ccc}
1 & 0 & 0 \\
0 & t & -\sqrt{1-t^{2}} \\
0 & \sqrt{1-t^{2}} & t
\end{array}\right) \\
& \left(\begin{array}{ccc}
\sqrt{\frac{-t}{1-t}} & \sqrt{\frac{1}{1-t}} & 0 \\
\sqrt{\frac{1}{1-t}} & -\sqrt{\frac{-t}{1-t}} & 0 \\
0 & 0 & 1
\end{array}\right)\left(\begin{array}{l}
1 \\
0 \\
0
\end{array}\right)=\left(\begin{array}{c}
0 \\
\sqrt{-t} \\
\sqrt{1+t}
\end{array}\right) .
\end{aligned}
$$

As $|0\rangle$ is the only accepted state, after the projective measurement on the final state, the QFA will accept the input string when its length is $k^{*} P$ and reject the string when its length is $k^{*} P+R$.

We now present our experimental demonstration of a QFA in linear optics. As shown in Fig. 2, a UV pulse laser with a central wavelength of $390 \mathrm{~nm}$, produced by second harmonic generation using Mira-HP (repetition rate of $76 \mathrm{MHz}$ and central wavelength of $780 \mathrm{~nm}$ ), is focused onto a barium borate (BBO) crystal cut for type-II spontaneous parametric down-conversion (SPDC) to generate a two-photon state. By detecting a horizontallypolarized single photon at detector D3, a vertically-polarized single photon on path $a\left(\left|V_{\mathrm{a}}\right\rangle\right)$ is obtained as the starting state of our QFA. We define $|0\rangle=\left|V_{\mathrm{a}}\right\rangle,|1\rangle=\left|H_{\mathrm{b}}\right\rangle$ and $|2\rangle=\left|V_{\mathrm{b}}\right\rangle$, where $H_{\mathrm{b}}$ $\left(V_{\mathrm{b}}\right)$ denotes the photon in horizontal (vertical) polarization on path $b$. The photon now encodes a qutrit and the starting state of the QFA is $|0\rangle$. To run the computation of the QFA, one needs to implement $n$ copies of $U$ on the qutrit, where $n$ is the length of the input string. As $U_{c}$ is simpler for experimental implementation, we will thus realize $U^{n}$ by cascading $U_{\mathrm{e}}, U_{c}^{n}$ and $U_{\mathrm{e}}^{\dagger}$. The qutrit photon first passes through the unitary $U_{\mathrm{e}}$, which is realized by the halfwaveplate HWP1 and the beam-displacer BD1, and the state is converted to $\sqrt{\frac{-t}{1-t}}|0\rangle+\sqrt{\frac{1}{1-t}}|1\rangle$. The photon then goes through a sequence of HWPs on path $b$. Every two consecutive HWPs (HWP2 and HWP3 for example) realize a $U_{c}$ which rotates the polarization state on path $b$ by an angle of $\frac{2 \pi Q}{P}$ and leaves the polarization on path $a$ unchanged. There are $n$ copies of $U_{c}$ along the path, where $n=k^{*} P$ or $k^{*} P+R$. After passing through $U_{c^{\prime}}^{n}$ the qutrit state would become $\sqrt{\frac{-t}{1-t}}|0\rangle+\sqrt{\frac{1}{1-t}}|1\rangle$ if $n=k * P$ or $\sqrt{\frac{-t}{1-t}}|0\rangle-$ $\sqrt{\frac{t^{2}}{1-t}}|1\rangle+\sqrt{1+t}|2\rangle$ if $n=k * P+R$. The qutrit photon further gets through HWP4 on path a (used to change $V_{\mathrm{a}}$ to $H_{\mathrm{a}}$ ), HWP5 on path $\mathrm{b}$ (used to swap $H_{\mathrm{b}}$ and $V_{\mathrm{b}}$ ) and BD2. These three optical devices together implement a unitary $M$ which maps $V_{\mathrm{a}}$ to $H_{\mathrm{b}}, H_{\mathrm{b}}$ to $V_{\mathrm{b}}$, and $V_{\mathrm{b}}$ to $H_{\mathrm{c}}$. After passing through $M$, the photon state can still be written as $\sqrt{\frac{-t}{1-t}}|0\rangle+\sqrt{\frac{1}{1-t}}|1\rangle$ or $\sqrt{\frac{-t}{1-t}}|0\rangle-\sqrt{\frac{t^{2}}{1-t}}|1\rangle+$ $\sqrt{1+t}|2\rangle$ if we redefine the basis states of the qutrit as $|0\rangle=\left|H_{\mathrm{b}}\right\rangle$, $|1\rangle=\left|V_{\mathrm{b}}\right\rangle$ and $|2\rangle=\left|H_{\mathrm{c}}\right\rangle$. At last, the qutrit photon passes through the unitary $U_{\mathrm{e}}^{\dagger}$, which is realized by HWP6, and the state is thus converted $|0\rangle=\left|H_{\mathrm{b}}\right\rangle$ or $\sqrt{-t}|1\rangle+\sqrt{1+t}|2\rangle=\sqrt{-t}\left|V_{\mathrm{b}}\right\rangle+\sqrt{1+t}$ $\left|H_{\mathrm{c}}\right\rangle$. The qutrit photon is then measured in the $|0 / 1 / 2\rangle$ basis and the string is accepted if the photon is detected at D0 or rejected if the photon is detected at D1 or D2.

To assess the performance of our experiment, we have configured our setup to realize 4 different QFAs. The first (second)
QFA is used to determine whether the length of the string is $3 k$ or $3 k+1(3 k+2)$. The third (fourth) QFA is used to determine whether the length of the string is $5 k$ or $5 k+1(5 k+2)$. As shown in Fig. 3a, b, for the first (second) QFA, the qutrit is projected to $|0\rangle$ with an average probability of $98.452 \pm 0.073 \%(98.516 \pm 0.087 \%)$ if the length of the string is 0,3 or $6(0$ or 3 ); If the length of the string is 1,4 , or 7 ( 2 or 5$)$, the qutrit is projected to $|1\rangle$ or $|2\rangle$ with an average probability $99.562 \pm 0.037 \%$ (99.634 $\pm 0.041 \%)$. Similarly, as shown in Fig. 3c, d, for the third (fourth) QFA, the qutrit is projected to $|0\rangle$ with an average probability of $98.697 \pm 0.080 \%$ $(98.711 \pm 0.079 \%)$ if the length of the string is 0 or $5(0$ or 5$)$; If the length of the string is 1 or 6 ( 2 or 7$)$, the qutrit is projected to $|1\rangle$ or $|2\rangle$ with an average probability of $99.461 \pm 0.052 \%(99.443 \pm$ $0.052 \%)$. The experimental results are all in good agreement with the theoretical predictions.

Our experimental setup can be used to distinguish much longer input strings by using more waveplates. As optical waveplates have very high transmission rate (>99.5\%), in principle we can use more than 1000 waveplates to implement the QFA, which can handle input strings longer than 500 characters. The main reasons prevent us from doing so are the high cost of a thousand waveplates and the limited size of the optical bench.

\section{DISCUSSION}

Here we highlight the key differences between DFA and QFA. By definition, in both DFA and QFA, the state transition will happen $n$ times, where $n$ is the length of the input string. As a result, there is no difference between DFA and QFA in time complexity. The key difference between DFA and QFA is in space complexity. For a DFA, the state inside is a classical system, which means all of its possible states are always orthogonal to each other. As a result, to distinguish between strings with the length of $k * P$ and $k * P+R$, the classical system inside the DFA must have a state space with at least $P$ orthonormal states. ${ }^{22,26}$ In contrast, for a QFA, the state inside is a quantum system, which means it can be in a superposition state and its possible states do not need to be orthogonal to each other. By utilizing this feature, the QFA with a state space of just three orthonormal states can be used to solve the same promise problem. Our proof-of-principle optical experiment have demonstrated this quantum advantage of QFA in space efficiency. By using a photonic quantum system with just three orthonormal states, our QFA solves a promise problem which would needs a DFA with at least $P$ orthonormal states.

Compared with DFA, Turing machine (classical computer) is a more general computing model equipped with memory. However, a Turing machine cannot solve the promise problem more space efficiently than a DFA. It would also need a state space with no less than $P$ orthonormal states to solve the same problem. To simulate a QFA solving the promise problem with a classical computer, the computer would needs an even larger state space (much larger than the state space with $P$ orthonormal states) to track the change of the amplitudes of the quantum state in the QFA.

In summary, we have experimentally constructed a proof-ofprinciple optical QFA, which can solve a particular promise problem-determining whether the length of an input string can be divided by a prime number $P$ with no remainder or with a remainder of $R$-with much higher space efficiency than its classical counterpart. To solve this problem, a classical DFA needs a state space with at least $P$ states, whereas a QFA only needs a quantum state space with three orthonormal states. The size of quantum state space for solving such problem is constant and does not depend on the value of $P$. Our results have clearly proven this quantum benefit of QFAs. However, in this work, the QFAs are just used to solve a special promise problem with unary alphabets. There are more interesting decision and promise problems using binary alphabets to be solved by QFAs. ${ }^{22,27}$ Our experiments pave 
the way for further research on using QFAs to tackle such problems.

\section{METHODS}

Experimental settings

As shown in Fig. 2, the setup are configured to realized four different QFAs to determine whether the length of an input string can be divided by $P$ with no remainder or with a remainder of $R$, where $(P, R)$ are set to be $(3,1)$, $(3,2),(5,1)$, or $(5,2)$. To realize these four QFAs, the half-waveplates are reconfigured to realize different $U_{\mathrm{e}}, U_{\mathrm{c}}$ and $U_{\mathrm{e}}^{\dagger}$. For the case of $(P, R)=(3,1)$ $((3,2),(5,1),(5,2))$, HWP1, HWP3, and HWP6 are set at $60^{\circ}\left(60^{\circ}, 72^{\circ}, 36^{\circ}\right)$, $27.37^{\circ}\left(27.37^{\circ}, 24.02^{\circ}, 24.02^{\circ}\right)$, and $60^{\circ}\left(60^{\circ}, 72^{\circ}, 36^{\circ}\right)$, respectively. For all the four cases, HWP2, HWP4, and HWP5 are always set at $0^{\circ}, 45^{\circ}$ and $45^{\circ}$ respectively.

\section{DATA AVAILABILITY}

The data that support this study are available from the corresponding author upon reasonable request.

\section{ACKNOWLEDGEMENTS}

This work was supported by the National Key Research and Development Program (2017YFA0305200 and 2016YFA0301700), the Key Research and Development Program of Guangdong Province of China (2018B030329001 and 2018B030325001) and the Natural Science Foundation of Guangdong Province of China (2016A030312012). S.Z. acknowledges supports from the National Natural Science Foundation of China (61602532) and the National Natural Science Foundation of Guangdong Province of China (2017A030313378). X.Z. acknowledges support from the National Young 1000 Talents Plan.

\section{AUTHOR CONTRIBUTIONS}

X.Z. and S.Z. devised the concept of the experiment. Y.T., T.F. and M.L. performed the experiment. X.Z., S.Z., Y.T. and T.F. performed the theoretical analysis. X.Z. managed the project. The manuscript was written by X.Z. and Y.T. with the input from all others. Y.T. and T.F. have contributed equally to this work.

\section{ADDITIONAL INFORMATION}

Competing interests: The authors declare no competing interests.

Publisher's note: Springer Nature remains neutral with regard to jurisdictional claims in published maps and institutional affiliations.

\section{REFERENCES}

1. Shor, P. W. Algorithms for quantum computation: discrete logarithms and factoring. In Proceedings of 35th Annual Symposium Foundations of Computer Science, 124-134 (IEEE, Santa Fe, NM, USA, 1994).

2. Feynman, R. P. Simulating physics with computers. Int. J. Theor. Phys. 21, 467-488 (1982).

3. Abrams, D. S. \& Lloyd, S. Simulation of many-body Fermi systems on a universal quantum computer. Phys. Rev. Lett. 79, 2586 (1997).

4. Aspuru-Guzik, A., Dutoi, A. D., Love, P. J. \& Head-Gordon, M. Simulated quantum computation of molecular energies. Science 309, 1704-1707 (2005).

5. Ladd, T. D. et al. Quantum computers. Nature 464, 45-53 (2010).
6. Lu, D. W. et al. Enhancing quantum control by bootstrapping a quantum processor of 12 qubits. Npj Quantum Inf. 3, 45 (2017).

7. Gambetta, J. M., Chow, J. M. \& Steffen, M. Building logical qubits in a superconducting quantum computing system. Npj Quantum Inf. 3, 2 (2017).

8. Figgatt, C. et al. Complete 3-qubit Grover search on a programmable quantum computer. Nat. Commun. 8, 1918 (2017).

9. Watson, T. F. et al. A programmable two-qubit quantum processor in silicon. Nature 555, 633-637 (2018).

10. Qiang, X. et al. Large-scale silicon quantum photonics implementing arbitrary two-qubit processing. Nat. Photonics 12, 534-539 (2018).

11. Debnath, $\mathrm{S}$. et al. Demonstration of a small programmable quantum computer with atomic qubits. Nature 536, 63-66 (2016).

12. Vandersypen, L. M. K. et al. Experimental realization of Shor's quantum factoring algorithm using nuclear magnetic resonance. Nature 414, 883-887 (2001).

13. Walther, P. et al. Experimental one-way quantum computing. Nature 434, 169-176 (2005).

14. Cai, X. D. et al. Experimental quantum computing to solve systems of linear equations. Phys. Rev. Lett. 110, 230501 (2013).

15. Li, Z., Liu, X., Xu, N. \& Du, J. Experimental realization of a quantum support vector machine. Phys. Rev. Lett. 114, 140504 (2015).

16. Wang, $\mathrm{H}$. et al. High-efficiency multiphoton boson sampling. Nat. Photonics 11, 361-365 (2017).

17. Hopcroft, J. E., Motwani, R. \& Ullman, J. D. Introduction to Automata Theory, Languages, and Computation. 3rd Edition (Addision-Wesley, Boston, 2006).

18. Culik, K. II. \& Kari, J. Image compression using weighted finite automata. Comput. Graph. 17, 305-313 (1993).

19. Kondacs, A. \& Watrous, J. On the power of quantum finite state automata. In Proceedings on 38th Annual Symposium Foundations of Computer Science, 66-75 (IEEE, Miami Beach, FL, USA, 1997).

20. Moore, C. \& Crutchfield, J. P. Quantum automata and quantum grammars. Theor. Comput. Sci. 237, 275-306 (2000).

21. Ambainis, A. Superlinear advantage for exact quantum algorithms. SIAM J. Comput. 45, 617-631 (2016). Earlier version in STOC'13.

22. Gruska, J., Qiu, D. W. \& Zheng, S. G. Potential of quantum finite automata with exact acceptance. Int. J. Found. Comput. Sci. 26, 381-398 (2015).

23. Eilenberg, S. Automata, Languages, and Machines (Academic Press, Orlando, 1974).

24. Yu, S. Handbook of Formal Languages, Regular Languages (Springer, Berlin, 1997).

25. Goldreich, O. Theoretical Computer Science-Essays in Memory of Shimon Even (Springer, Heidelberg, 2006).

26. Ambainis, A. \& Yakaryılmaz, A. Superiority of exact quantum automata for promise problems. Inform. Process. Lett. 112, 289-291 (2012).

27. Ambainis, A. \& Watrous, J. Two-way finite automata with quantum and classical states. Theor. Comput. Sci. 287, 299-311 (2002).

cc Open Access This article is licensed under a Creative Commons Attribution 4.0 International License, which permits use, sharing, adaptation, distribution and reproduction in any medium or format, as long as you give appropriate credit to the original author(s) and the source, provide a link to the Creative Commons license, and indicate if changes were made. The images or other third party material in this article are included in the article's Creative Commons license, unless indicated otherwise in a credit line to the material. If material is not included in the article's Creative Commons license and your intended use is not permitted by statutory regulation or exceeds the permitted use, you will need to obtain permission directly from the copyright holder. To view a copy of this license, visit http://creativecommons org/licenses/by/4.0/.

(c) The Author(s) 2019 